\title{
Numerical analysis of capillary bridges and coalescence in a triplet of spheres.
}

\author{
Marie Miot · Guillaume Veylon · Antoine \\ Wautier · Pierre Philippe · François Nicot •
}

Frédéric Jamin

Received: date / Accepted: date

\begin{abstract}
The behavior of a natural soil is known to change substantially in presence of water under unsaturated conditions, due to additional capillary forces. Water can be absorbed by hygroscopic soil particles (such as clay), or remains at the surface of solid grains (sand, silt) and forms either a discontinuous (pendular regime) or a continuous phase (funicular regime), depending on the water content of the soil. Capillary bridges exist solely between pairs of grains at small water contents, giving rise to simple capillary force expressions and straightforward subsequent modeling. For larger water contents, these generic capillary bridges
\end{abstract}

M. Miot

INRAE, Aix Marseille Univ., RECOVER, 3275 Rte Cézanne, CS 40061, 13182 Aix-en-Provence Cedex 5, France

E-mail: marie.miot@inrae.fr

G. Veylon

INRAE, Aix Marseille Univ., RECOVER, 3275 Rte Cézanne, CS 40061, 13182 Aix-en-Provence Cedex 5, France

A. Wautier

INRAE, Aix Marseille Univ., RECOVER, 3275 Rte Cézanne, CS 40061, 13182 Aix-en-Provence Cedex 5, France

P. Philippe

INRAE, Aix Marseille Univ., RECOVER, 3275 Rte Cézanne, CS 40061, 13182 Aix-en-Provence Cedex 5, France

F. Nicot

Université Grenoble Alpes, INRAE, ETNA, 2 Rue de la Papeterie BP 76, 38402 Saint Martin d'Hères, France

F. Jamin

LMGC, Univ. Montpellier, CNRS, Montpellier, France, 860 Rue de St - Priest, 34090 Montpellier, France 
progressively merge into more complex coalesced bridges involving several grains (i.e. at least three) and whose description remains little known. In the present study, a numerical approach based on surface energy minimization is proposed to compute capillary forces for assemblies of two or three grains. The methodology is first validated for a standard capillary bridge between two grains by comparison both with previous experiments and with other alternative theoretical and numerical approaches. The method is next extended to a triplet of grains within a wide range of water content (or equivalently reduced water volume) during imbibition, to switch from uncoalesced to coalesced bridges. Eventually, the influence of contact angle, surface tension and gravity on the capillary force, the volume of coalescence and the morphology of the bridge as well is investigated. The present study paves the way for the implementation of capillary effects in micromechanical models relying on mesostructures composed of a few grains.

Keywords Capillary forces · Coalescence · Surface energy · Unsaturated conditions · Granular materials

\section{Introduction}

2 Granular material is involved in a great variety of phenomena and processes from natural hazards to industrial applications. Although intensively modeled for the sake of simplicity as dry grains interacting solely through frictional forces at contacts, a large class of granular materials exhibits substantial capillary effects depending on both the size of the constitutive particles and the water content. A famous example in everyday life is the sand castle on the beach, whose mechanical stability relies on an optimal water content $[1,2]$. In the field of civil engineering, the mechanical behavior of unsaturated granular materials is of particular interest when considering earthen dykes or dams, where different regimes of water saturation can coexist. A fully saturated material is mainly equivalent to a dry material, owing that effective stresses $\sigma^{\prime}$ are introduced using Terzaghi's relationship [3]: $\underline{\underline{\sigma}}^{\prime}=\underline{\underline{\sigma}}-u_{w} \underline{\underline{I}}$, with $u_{w}$ the pore water pressure. In the case of partially saturated media, capillary effects give rise to internal stresses (often referred to as suction) within the material, inducing significant changes in its mechanical behavior [4] and

16 Therzaghi's relationship does not hold anymore. The complexity of unsaturated 

44 grains.

granular materials stems from the existence of different capillary regimes [5-7], depending on the degree of saturation, the pore geometry and the wettability.

Apart from the specific case of hygroscopic materials, such as clay, where water is adsorbed by the outer surface of the porous particles (so-called hygroscopic regime), the capillary regimes in a material made up of solid particles are commonly described as follows. For small enough water contents, water spontaneously forms bridges connecting two or more grains but with overall water phase remaining discontinuous. This corresponds to the pendular regime. When water content increases, the progressive coalescence of all bridges results in a continuous liquid phase and the material is said to be in the funicular regime [5]. This regime remains challenging to be modeled numerically at the sample scale: in DEM, only the pendular regime has been implemented [8,9], and the Lattice Boltzmann Method, that can essentially model any capillary regime, is computationally expensive [10-12]: for instance, modeling of water drainage in a 40-grains packing takes more than 100 days. Funicular regime is mostly studied experimentally, for example in triaxial tests in Bishop et al. [13] and in shear tests in Cuomo et al. [14].

Many previous studies have focused at the local scale on capillary bridges between a few grains in the pendular regime. The shape of an axisymmetric capillary bridge between a pair of grains and the resulting axial capillary force have been studied experimentally [15-17], and theoretically, using Laplace-Young equation, with either cylindrical [18], toroidal [19-21], or elliptic [22] approximations of the bridge profile. A mixed methodology is presented in [23-25], coupling experiments, through accurate measurements of the bridge geometry, and numerical resolution of Laplace-Young equation in order to evaluate capillary pressure and force. At the macroscale, for small degrees of saturation (typically less than 10\%), capillary forces can be accounted for with a discrete element method, both in static $[9,26-29]$ and dynamic situations [30-32], considering solely liquid bridges between pairs of

Hysteresis effects on the capillary forces and on the shape of water bridge at microscale have been measured experimentally [33]. These effects can be attributed to the hysteretic behavior of the contact angle [34] and to the existence of multiple morphologies for a liquid drop in contact with more than two grains, as it was underlined in the case of a triplet of spheres in Semprebon et al. [35]. Coalescence and 
rupture of water bridges in assemblies of more than two grains also lead to a hysteresis in the evolution of the capillary forces. Coalescence of axisymmetric bridges between pairs of grains into more complex bridges in contact with more than two grains has been studied experimentally $[36,37]$. Experimental desiccation of water in assemblies of 3 grains and more was performed in [38]. Numerically, the coalescence of three bridges between equidistant spheres has been considered by solving the Laplace-Young equation both in 2D [39,40] and in 3D [41], and the resulting coalesced bridge was studied in [42]. The rupture of a coalesced bridge into two separate pendular bridges was studied using an energy minimization method [43]. However, more complex assemblies are difficult to handle directly through the Laplace-Young equation because of the lack of symmetry and advanced numerical methods are needed [10-12].

In this paper, we propose an alternative advanced method (computationally less expensive) to compute capillary forces in an assembly of three grains, based on an energy minimization approach. To this respect, the energy minimization software Surface Evolver is used [44]. This method has been implemented to solve different problems, such as capillary bridges between two planes [45] or nonsymmetric capillary bridges [46]. The main benefit of this method is that the energy minimization approach provides the geometrical shape of capillary bridges, for any grain configurations and for any given water volume, knowing the contact angle. This method is also truly efficient to include gravity effect on the morphology of a bridge. However, it does not account automatically for coalescence and rupture of bridges. This method has already been used to model water bridges between more than two grains [43], but to the best of our knowledge, no study about the influence of contact angle and gravity on the coalescence of two capillary bridges in a triplet of grains has been carried out so far. In this paper, a comparison between an experimental study of the coalescence of two capillary bridges in a triplet of grains by El Korchi et al. [37] and numerical results obtained from energy minimization provides the opportunity to capture the effect of surface tension, contact angle and gravity on the capillary force, the volume of coalescence and the morphology of the coalesced bridge. The present results pave the way for the development of enriched micromechanical models in which the constitutive behavior is deduced from the behavior of a collection of mesostructures composed 
of a few grains. In particular, the method seems to be perfectly adapted for the calculation of the capillary forces in the ten-grain mesostructure of the so-called H-model [47].

This paper is organized as follows. We first present the energy method used to compute the capillary forces in an assembly of grains. This approach is next validated for an axisymmetric capillary bridge between two grains, by comparing the results with an exact numerical solution [9] and related empirical approximation $[16,26]$, an analytical expression based on a cylindrical approximation of the bridge [18] and several experimental results [48]. Capillary forces calculations are then performed on an assembly of three grains with two merging capillary bridges. The results are compared with the experimental results obtained by El Korchi et al. [37] and eventually discussed, including detailed analyses on the influences of the main numerical parameters such as contact angle, surface tension and gravity.

\section{Energy method for capillary forces calculation}

\subsection{Capillary forces calculation}

The capillary bridges and related forces for a given geometric arrangement of spherical grains and a given volume of water are calculated by means of a surface energy minimization method, using the open source software Surface Evolver [44].

For an unsaturated granular assembly composed of $\mathrm{N}$ grains, the surface energy $E_{s}$ is given by:

$$
E_{s}=\gamma^{l g} A^{l g}+\sum_{i=1}^{N} \gamma_{i}^{s l} A_{i}^{s l}+\sum_{i=1}^{N} \gamma_{i}^{s g} A_{i}^{s g}
$$

where $\gamma^{l g}$ is the surface tension and $A^{l g}$ the area of the liquid/gas (i.e. water/air) interface, $\gamma_{i}^{s l}$ and $A_{i}^{s l}$ as well as $\gamma_{i}^{s g}$ and $A_{i}^{s g}$ are respectively the surface tension and the area of the interface between grain $i$ and liquid (i.e. water), and between grain $i$ and gas (i.e. air).

In addition, the surface tensions of the different interfaces are related by the Young-Dupré equation [49]: 


$$
\gamma_{i}^{s l}-\gamma_{i}^{s g}=-\cos \theta_{i} \gamma^{l g}
$$

where $\theta_{i}$ stands for the contact angle of water on grain $i$.

Thus, the surface energy of the system can be expressed as follows:

$$
E_{s}=\gamma^{l g} A^{l g}-\gamma^{l g} \sum_{i=1}^{N} \cos \theta_{i} A_{i}^{s l}+C
$$

where $C=\sum_{i=1}^{N} \gamma_{i}^{s g} A_{i}^{s}$ is a constant for a given grain assembly, with $A_{i}^{s}=A_{i}^{s g}+$ $A_{i}^{s l}$ the total area of grain $i$. Eventually, it appears from Eq. (3) that the variation in surface energy depends solely on the overall geometry of the liquid interfaces.

The geometry of the liquid interfaces minimizing surface energy is computed using the gradient descent method implemented in Surface Evolver. As energies are usually defined up to a constant, only energy differences are tracked for the capillary force calculation. The constant $C$ is thus not calculated and left aside, which means that the solid/gas interfaces do not need to be modeled. Consequently, the problem to be solved is an energy minimization calculation on the liquid phase under constraints on the positions of solid/liquid interfaces and on the liquid volume. The specificity of this gradient descent method concerns its application on a mesh of the liquid phase interfaces that is refined several times during the calculation [44]. As a result, the number of degrees of freedom handled in the gradient descent steps regularly increases during the process.

Figure 1 illustrates the different steps of the surface energy minimization scheme. First, a basic geometry with few vertices and an arbitrary volume is defined. Then, many iterations of gradient descents are carried out while remeshing is performed periodically, in order to increase progressively the number of vertices and improve the precision of the calculation. During remeshing phases, the size distribution of edges lengths is kept small enough by removing and refining short and long edges, respectively [35]. The iterative numerical process is thus a succession of energy minimization and remeshing steps.

Once the optimized geometry is reached, capillary forces acting on a given grain assembly are determined relying on the Virtual Work Principle. Note that, even though the capillary force acting on a grain could also be calculated with 


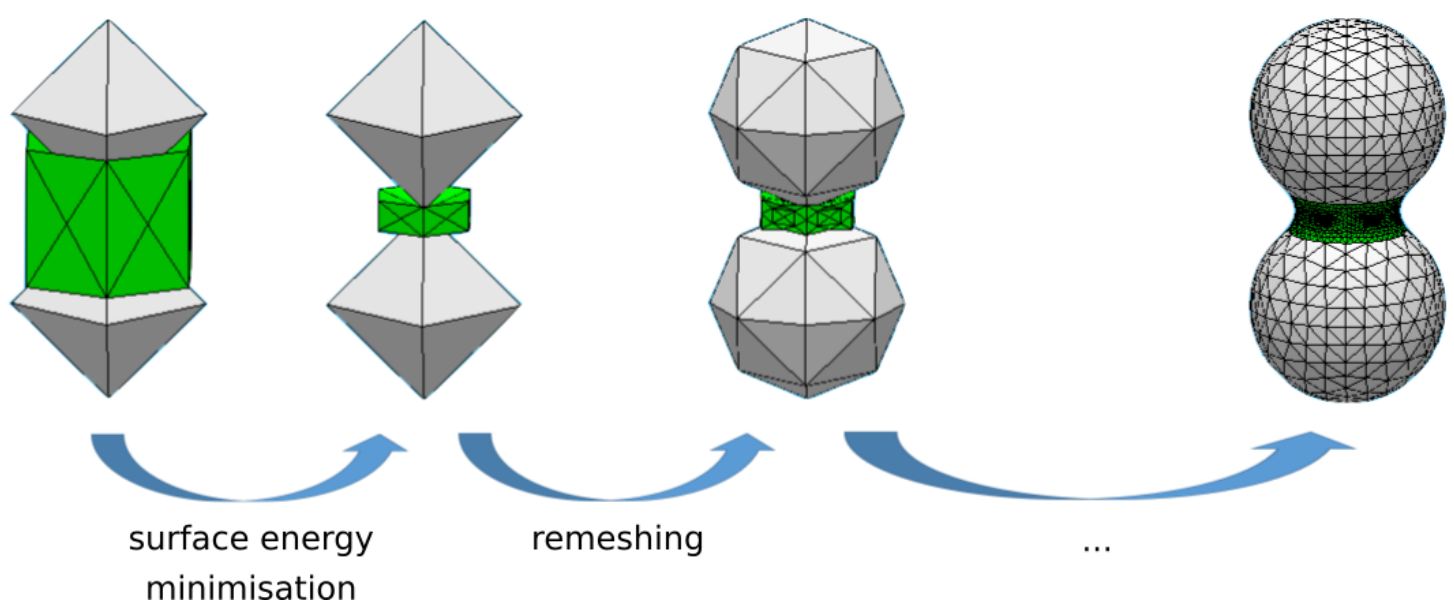

Fig. 1 Illustration of the energy minimization procedure scheme for a capillary bridge between two grains from the initial to the optimized geometry Note that the grains are plotted only for the sake of illustration. The grain surface is accounted for by a geometric constraint in the Lagrangian formulation of the problem.

the boundary method [35], the Virtual Work Principle provides directly the external forces of a system of several grains with capillary bridges. The formalism obtained with this method is thus well adapted to account for capillary effects in micromechanical models as for instance the $\mathrm{H}$-model relying on mesostructures of 10 grains [47]. Such a work is currently under development [50]. The system is consequently stretched in a given direction corresponding to a virtual incremental displacement $\overrightarrow{\delta d}$ (Figure 2), and the surface energy is estimated independently in both initial and stretched configurations. Assuming that the system is closed (no change in water volume), static (no kinetic energy) and non-dissipative, the incremental work $\delta W$ of the external force $\overrightarrow{F_{c}}$ can be expressed as:

$$
\delta W=\overrightarrow{F_{c}} \cdot \overrightarrow{d d}=\delta E_{s}+\delta E_{p}
$$

with $\delta E_{s}$ the incremental variation in surface energy between the two configurations and $\delta E_{p}$ the incremental variation in potential energy. Here, only potential energy due to gravity can be optionally considered (see Section 3.4).

The capillary force component acting in an arbitrary direction $\vec{u}$ is then directly related to the variation of the surface energy and the incremental displacement, and reads: 


$$
\overrightarrow{F_{c}} \cdot \vec{u}=\frac{\delta E_{s}+\delta E_{p}}{\overrightarrow{\delta d} \cdot \vec{u}}
$$

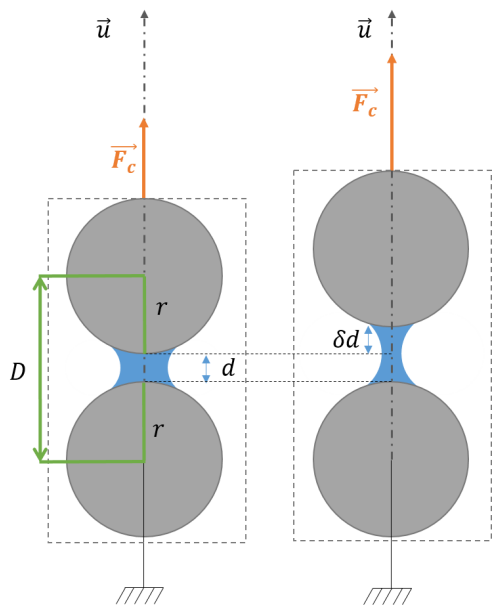

Fig. 2 Determination of a capillary force along direction $\vec{u}$ with the Virtual Work Principle.

Compared with standard energy minimization techniques performed over a fixed number of degrees of freedom, the main difficulty of the present procedure lies in the selection of an optimal number of gradient descent steps between two successive remeshing steps in order to prevent a stalling of gradient descent method due to a too small scale factor. The choice in $\overrightarrow{\delta d}$ should allow energy variations larger than numerical noise but small enough for non-linear effects to be neglected. A parametric study is thus necessary to determine the most efficient parameters for any given grain assembly. An example of such a parametric study is given in Appendix A for the triplet of grains considered in the forthcoming Section 3, with restriction to $\theta=0^{\circ}$.

2.2 Capillary force in a single bridge between two grains

The purpose of this subsection is to check the validity of the numerical protocol based on the energy method presented in the previous section. The most standard example of an axisymmetric liquid bridge connecting two spheres is modeled. This 
very simple benchmark case was widely discussed in the literature [15-25]. More complex configurations will be addressed thereafter.

Thus, we consider here two spherical grains with the same radius $r$ and the same contact angle with liquid, namely $\theta=0^{\circ}$. The distance between the centers of the spheres is $D=2 r+d$, with $d$ the intergranular distance. A given volume $V$ of liquid is entirely used to form a capillary bridge connecting the two grains. Because the geometry of the capillary doublet is left invariant by rotation around the axis joining the two grain centers, the axisymmetric liquid bridge generates a capillary force $F_{c}$ along this direction denoted by $\vec{u}$ (Figure 2). All the quantities of interest are normalized with the radius and the surface tension of the liquid/gas interface $\gamma^{l g}$ and the star superscript refers to dimensionless quantities in the following. Accordingly, the dimensionless capillary force $F_{c}^{*}=F_{c} /\left(2 \pi \gamma^{l g} r\right)$ in the axial direction $\vec{u}$ is calculated as a function of the dimensionless intergranular distance $d^{*}=d / r$, for different dimensionless volumes of water $V^{*}=V / r^{3}$.

The results from this energy minimization method are compared to some previous studies published in the literature. Since the capillary bridge has axial symmetry, the Laplace-Young equation can be solved numerically [9], or analytically with a cylindrical approximation. Cylindrical approximation leads to a straightforward relationship between the capillary force and the intergranular distance [18]:

$$
F_{c}^{*}=\cos \theta\left(1-\frac{1}{\sqrt{1+\frac{2 V^{*}}{\pi d^{*} 2}}}\right)
$$

This relationship is only valid for small volumes of water, typically for $V^{*}<$ 0.01. An expression has also been proposed by Richefeu et al. [26] in order to fit the numerical solution of Laplace-Young equation in monodisperse condition (i.e. grains of the same diameter):

$$
F_{c}^{*}=\cos \theta \exp \left(\frac{-d^{*}}{0.9 \sqrt{V^{*}}}\right)
$$

Another more complex and precise expression obtained with an approximation of the numerical solution of Laplace-Young equation is provided by Willett et al. [16]. Moreover, this relationship is valid for a larger range of volumes than equations (6) and (7) are, namely for $V^{*}<0.1$. 
The expressions of the dimensionless capillary force obtained with the different models are plotted in Figure 3, as a function of the dimensionless intergranular distance for two values of dimensionless water volume: $V^{*}=0.008$ and $V^{*}=0.156$, which had been used in the experiments realized by Mielniczuk et al. [48]. In all cases, the capillary force decreases with the intergranular distance, except in the experiments with the highest water volume where a slight increase is observed at very small intergranular distances, namely $d^{*}<0.05$.

The capillary force deduced from surface energy minimization matches fairly well with the numerical solution of Laplace-Young equation [9] and the expression proposed by Willett et al. [16]. This agreement can be partly justified from the equivalence between resolution of Laplace-Young equation and surface energy minimization (as implemented in Surface Evolver) which has been discussed in this particular case in Lambert et al. [51]. The obtained results are also in good agreement with the experimental results [48], even if the capillary force obtained from the energy method is systematically higher for the small intergranular distances, especially for the large dimensionless volume. It is worth noting that the differences between numerical and experimental results at low intergranular distances can have two possible explanations. First, for low intergranular distances, the optimized incremental displacement $\delta d$ must be sufficiently large to induce a significant variation of energy, but smaller than the intergranular distance, in order to avoid having an interpenetration of the grains. Secondly, in the experiments, for low intergranular distance, a contact between grains is possible, which could substantially affect the measurement of the capillary force with the laboratory balance. Finally, the results obtained from the energy minimization method agree also fairly well with the analytical equations (6) and (7), demonstrating the good ability to predict experimental and numerical results over a wide range of water volumes.

\section{Capillary forces in an assembly of three spheres}

This section is devoted to (i) an extension of the model to calculate capillary forces for higher volumes of water involving larger grain assemblies, and (ii) a practical way to account for the possible coalescence of capillary bridges. Indeed, 

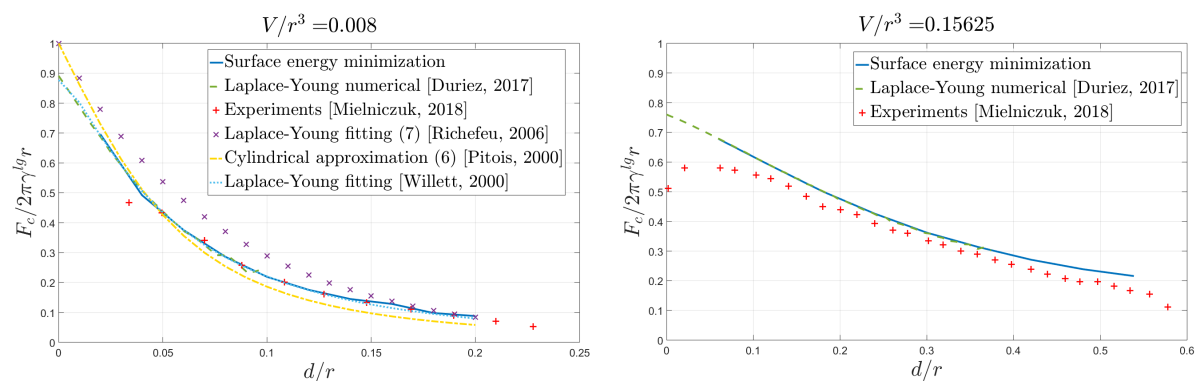

Fig. 3 Dimensionless capillary forces in a liquid bridge, as a function of the dimensionless intergranular distance, obtained with Surface Evolver (blue solid line), by solving Laplace-Young equation numerically (green dashed line) or analytically with a cylindrical approximation (yellow dash-dot line), with fittings of Laplace-Young relationship provided by Richefeu et al. [26] (crosses) and by Willett et al. [16] (light blue dotted line), and experimentally (red plus symbols). Note that the cylindrical approximation cannot be plotted for $V^{*}>0.01$ and fittings of Laplace-Young equation for $V^{*}>0.1$.

when a capillary bridge connects more than two grains, the axisymmetry of the geometry is usually broken. Such a lack of symmetry makes the resolution of Laplace-Young equation far more complex, underlying the relevance of the surface energy minimization method. The methodology presented in the previous section is thus extended here to analyze the coalescence of two liquid bridges in a triplet of grains, taking the opportunity of a direct comparison with the experimental results from [37]. The objectives are to show the ability of the numerical procedure to handle complex grain configurations for a wide range of water volume and to analyze the influences of several physical parameters.

\subsection{Description of the experiments}

The experimental set-up developed in [37] consists of a triplet configuration with three identical spherical glass beads of the same radius $r=4 \mathrm{~mm}$. The base of the assembly is constituted of two beads, the centers of which are separated by a distance $D_{2}=8.3 \mathrm{~mm}$. The third bead is placed above, in the median plane, at a distance $D_{1}=8.7 \mathrm{~mm}$ from the centers of the other beads as presented in Figure 4. Two capillary bridges between the upper bead and each of the two lower beads are initially created, using a micro-syringe. Then water is progressively added by steps of $2 \mu \mathrm{l}$ in each bridge until they merge. Afterward, water is added by steps of $4 \mu \mathrm{l}$ in the coalesced bridge. 
The vertical capillary force is measured by differential weighing of the system composed of the volume of water and the two lower beads. Indeed, while the upper bead remains fixed, the two lower ones lie on a precision scale. If water bridges were not attached to the upper bead, the scale would measure the mass of water plus the mass of the beads. In practice, this maximal available force is reduced by the capillary force exerted on the upper bead and the vertical capillary force is thus deduced from the difference between the theoretical maximal weight and the actual measurement.

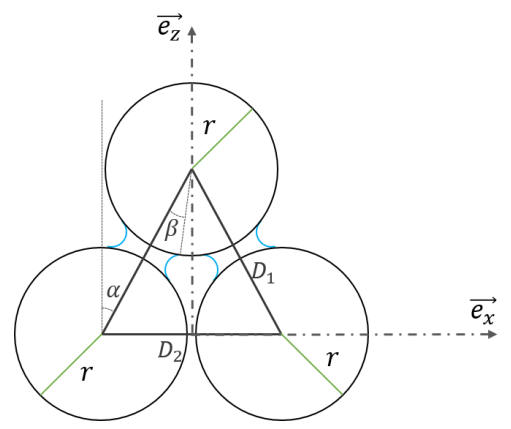

Fig. 4 Geometry of the triplet of grains with definition of both the half-filling angle $\beta$ of a bridge and the opening angle $\alpha$.

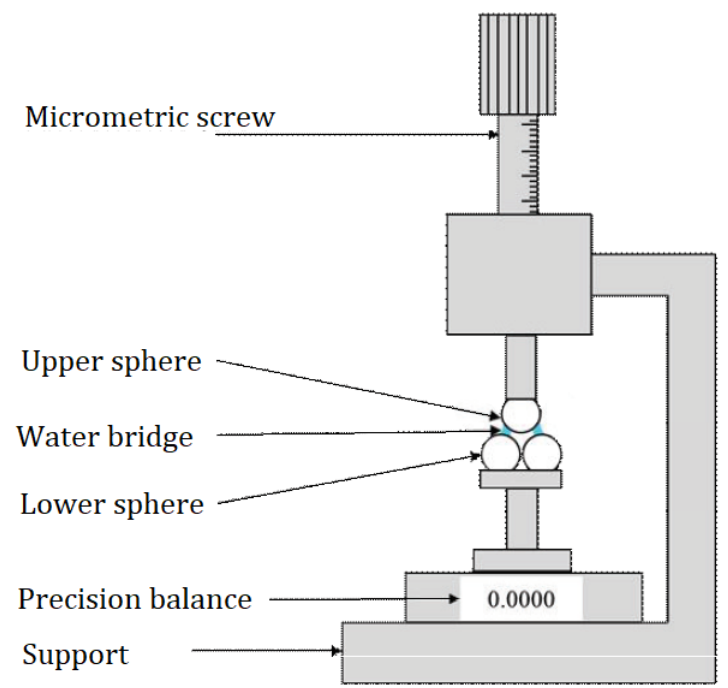

Fig. 5 Scheme of the experimental set-up from [37]. 
3.2 Modeling of imbibition of a triplet of grains

In order to model the experiment presented above, the standard value of the surface tension between pure water and air at $20^{\circ} \mathrm{C}$ is first selected, namely $\gamma^{l g}=$ $0.073 \mathrm{~N} / \mathrm{m}[52]$. The contact angle $\theta$ is taken as zero and gravity is not considered as a preliminary approximation. The relative influence of these different control parameters will be discussed later, in sections 3.3 and 3.4 .

For low volumes of water, two inclined capillary bridges exist between the upper sphere and each of the two other spheres. Consequently, the vertical capillary force is directly deduced from the previous results obtained for a capillary bridge between two grains (Section 2.2) by simple addition of the vertical components of the axial capillary force of the two inclined bridges. This approach remains valid as long as the two inclined bridges in Figure 4 do not merge. As the energetic approach is not capable of predicting merging of water volumes, a geometrical criterion is alternatively proposed in order to evaluate the volume corresponding to the merging of the two capillary bridges into a unique coalesced bridge. The half-filling angle $\beta^{1}$ of the bridge, as defined in Figure 4, is measured in order to detect the volume $V_{\text {coal }}$ for which $\beta=\alpha$ [39]. At this point, the water in the two bridges forms a common volume, which will evolve to a substantially different geometric configuration when minimizing the surface energy. For $V \geqslant V_{\text {coal }}$, the coalesced bridge is modeled using the numerical parameters determined in the parametric study presented in Appendix A.

In Figure 6, the results, obtained first for two uncoalesced bridges and second for a unique coalesced bridge, are compared with the experimental results. The latter shows an increase in the capillary force as a function of the water volume added in the two capillary bridges. The force increase gets progressively smaller until a plateau is almost reached. Coalescence of the two bridges occurs between $16 \mu \mathrm{l}$ and $20 \mu \mathrm{l}$, together with a substantial increase in the capillary force. For water volumes greater than $24 \mu \mathrm{l}$, when water is added in the coalesced bridge, the capillary force is found to decrease slightly.

1 In the literature, the half-filling angle is generally denoted $\delta$, but, in order to avoid any confusion with the symbol used before for infinitesimal variation, it is here denoted $\beta$. 


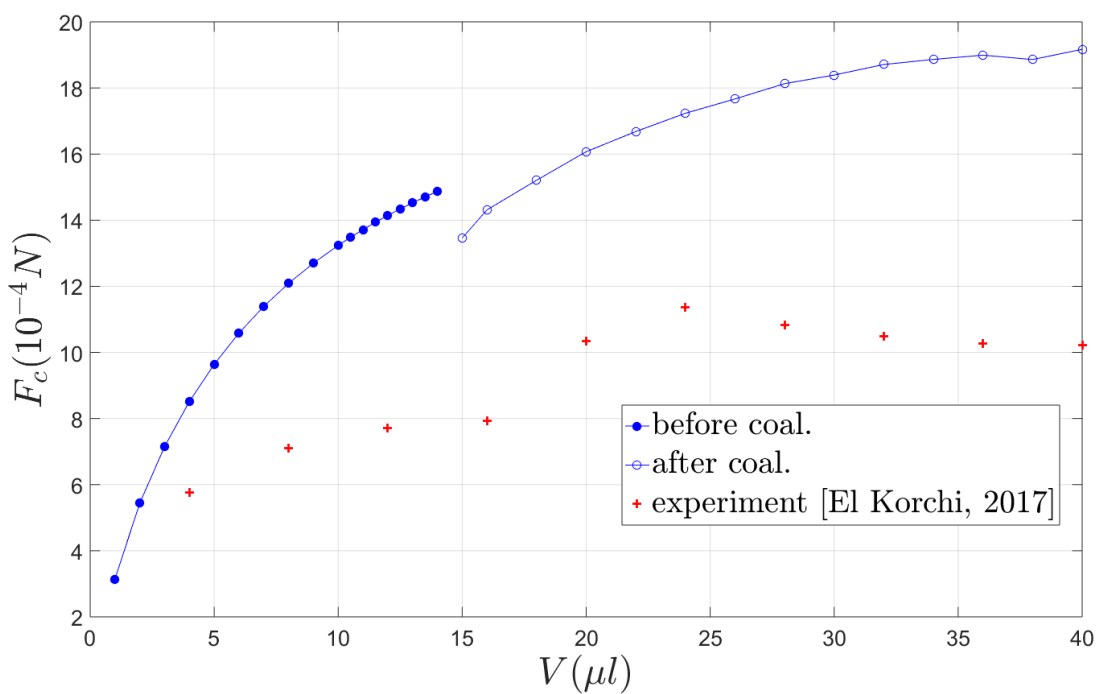

Fig. 6 Capillary force in a triplet of spheres, as a function of the injected volume of water. Numerical results are obtained with Surface Evolver for a contact angle $\theta=0^{\circ}$, with the standard surface tension value $\gamma^{l g}=0.073 \mathrm{~N} . \mathrm{m}^{-1}$. The solid and open symbols stand for the uncoalesced (two bridges) and coalesced (single cluster) regimes, respectively. The experimental data (red plus symbols) are taken from [37] (plus symbols). Coalescence occurs for $V$ between $14 \mu \mathrm{l}$ and $15 \mu \mathrm{l}$ with the numerical method, and for $V$ between $16 \mu \mathrm{l}$ and $20 \mu \mathrm{l}$ in the experiment.

In the numerical simulations with the standard values chosen for the control parameters, the capillary force also increases until the geometrical coalescence criterion is met, for a total volume between $14 \mu \mathrm{l}$ and $14.5 \mu \mathrm{l}$. However, several major differences must be pointed out. First, the values of the capillary force are systematically about 1.5 to 2 times larger than the experimental values. Secondly, the capillary force is found to drop at coalescence and not to increase as measured. Finally, the capillary force further increases at larger volumes, presumably tending to a plateau.

Although the proposed energetic approach has been validated in previous section for the modeling of a simple liquid bridge between a pair of spherical grains, it clearly fails to predict quantitatively the experimental data with standard parameter values, even in the uncoalesced regime. This suggests that the effective values of the main physical parameters (such as surface tension and contact angle) may differ from the classically admitted values, namely $\gamma^{l g}=0.073 \mathrm{~N} . \mathrm{m}^{-1}$ at $20^{\circ} \mathrm{C}$ 
(see for instance Molenkamp and Nazemi [52]) and $\theta \leqslant 10^{\circ}$ for glass beads (see for instance Scheel et al. [53], or Duriez and Wan [9]) and that gravity needs to be taken into account. Further investigations on the influence of these parameters, including gravity, are thus carried out in the following sections.

\subsection{Influence of the contact angle}

Although contact angle is a key parameter in all capillary phenomena, an experimental measure of its value is complex. Pictures of the experiments presented in [37] show that the contact angle changes with the volume of water and the position of the triple line (i.e. the intersection of liquid, gaseous and solid interfaces). The roughness and the cleanliness of the beads surface are known to affect locally its value, as well as the presence of adsorbed water at the solid surface. By way of illustration, in a bridge between two grains separated by $0.7 \mathrm{~mm}$, with a radius of $8 \mathrm{~mm}$ and a volume of $1 \mu \mathrm{l}, 4 \mu \mathrm{l}$ and $10 \mu \mathrm{l}$, the contact angle may vary between $7.2^{\circ}$ and $13.7^{\circ}$, according to [48]. In a triplet of grains, the contact angle of a water coalesced bridge was measured in Wang et al. [43] with values ranging between $10^{\circ}$ and $70^{\circ}$. In the coalesced domain, the pictures of the experiment show that the water cluster can even become convex along particular triple lines. This is visible for instance in Figure 7, where a contact angle greater than $90^{\circ}$ can be observed. Figure 7 depicts the profile of water interfaces before and after coalescence in both numerical simulations and experiments. After coalescence, part of the triple lines lies on surfaces that were previously covered by water before coalescence (the two interfaces between the top and bottom grains in the 2D cut in Figure 7), while some other portions of the triple line lie on surfaces that were dry before coalescence (the interfaces between the bottom grains in the $2 \mathrm{D}$ cut in Figure 7 ). The past state and history of the surface (wet or dry) are thus likely to generate large heterogeneities in contact angle at the surface of a same grain.

It is also worth pointing out a striking difference in the geometry of the coalesced bridge, the lower meniscus of which is located above the horizontal line joining the centers of the two bottom grains in the experiments, forming a so-called dimmer [35] whereas it is located below this line in the simulations presented in this manuscript, forming a so-called trimmer. As depicted in Figure 8, a lower po- 

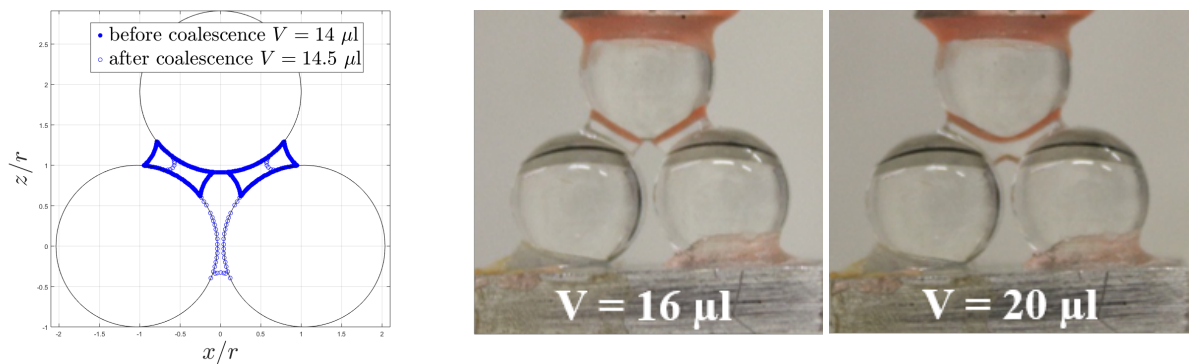

Fig. 7 Profile of water interfaces before and after coalescence. Left: position of the interfaces obtained numerically, in the plane formed by the centers of the grains just before coalescence for $V=14 \mu \mathrm{l}$ (full circles) and just after coalescence for $V=14.5 \mu \mathrm{l}$ (empty circles)). Middle and right: photographs of the water between the three grains in the experiment [37], before coalescence for $V=16 \mu \mathrm{l}$ and after coalescence for $V=20 \mu \mathrm{l}$.

sition of the meniscus will increase the horizontal component of the capillary force and decrease the vertical one. Moreover, a dimmer instead of a trimmer configuration tends to concentrate the volume of water in the upper part of the coalesced bridge, which increases in return the radius of curvature of the upper meniscus and then the vertical capillary force, according to [54]. Consequently, the vertical capillary force increases after coalescence in the experiments while it decreases in the simulations. For the present geometric configuration, no static equilibrium in a dimmer configuration was found. This suggests that the dimmer configuration observed experimentally is a metastable configuration.

a)

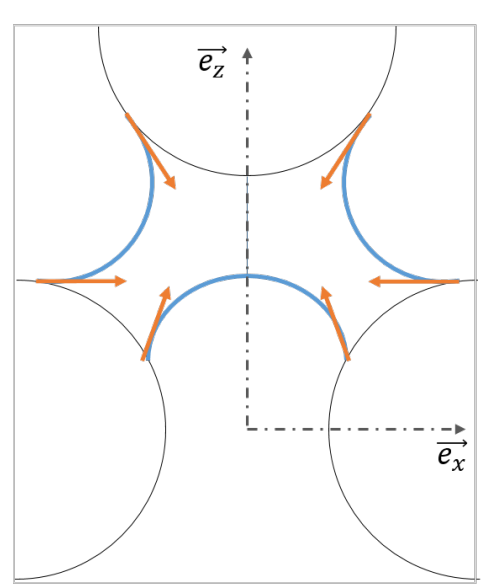

b)

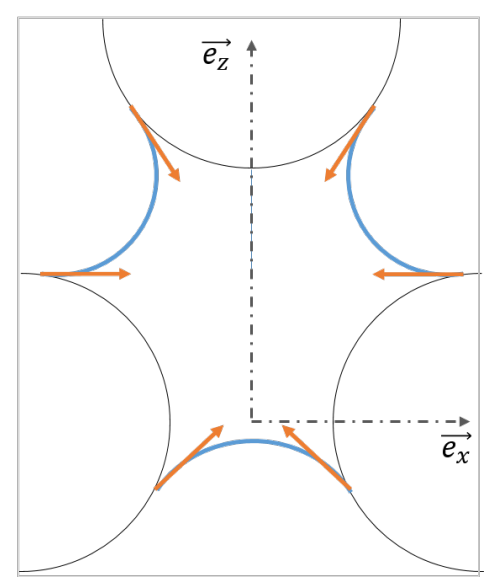

Fig. 8 Schemes of the influence of the meniscus position on the capillary force components: a) for a lower meniscus above the centers of the lower grains. b) For a lower meniscus below the centers of the lower grains. 
Figure 9 shows the capillary force acting on the top grain of the triplet as a function of the volume for a large range of contact angles. From $\theta \geqslant 30^{\circ}$, the capillary force no longer decreases but starts to increase at the coalescence transition, from two liquid bridges to a single capillary cluster. Keeping the standard value $\gamma^{l g}=0.073 \mathrm{~N} . \mathrm{m}^{-1}$, the numerical curve approximately fits the experimental curve before coalescence for $\theta \simeq 60^{\circ}$. After coalescence, the experimental curve lays between the numerical curves obtained for $\theta=50^{\circ}$ and $\theta=60^{\circ}$, which seems plausible according to [43] and to the pictures of the experiments presented in Figure 7. As mentioned previously, it is known that the contact angle depends on whether the surface was previously wet or dry. Based on the observations in Figure 7 , a change in the contact angle is thus physically relevant.

In addition to the change in capillary force at coalescence, Figure 10 also highlights that the total volume of water at coalescence $V_{\text {coal }}$ increases with the contact angle, perfectly linearly:

$$
V_{\text {coal }}=V_{\text {coal }}^{0}+\frac{\theta}{\Delta \theta} \Delta V_{\text {coal }}
$$

with $V_{\text {coal }}^{0}=14.5 \mu \mathrm{l}, \Delta V_{\text {coal }}=18 \mu \mathrm{l}$ and $\Delta \theta=90^{\circ}$.

Such a linear relationship is reminiscent of the one observed between the rupture distance and the contact angle in a capillary bridge between two grains, proposed by Lian et al. [20]. However, these similar behaviors are most probably coincidental or fortuitous since the two criteria of regime change do not have the same origin. The rupture distance in a capillary bridge is considered during drying or when intergranular distances increase, and depends on the surface energy of the liquid bridge. On the other hand, the volume of coalescence of capillary bridges has to be evaluated during imbibition or when intergranular distances decreases, and depends on the geometry of the grains assembly.

\subsection{Influence of gravity}

The effect of gravity can also partly explain the difference between experimental and numerical capillary forces. Indeed, gravity tends to deform the two inclined bridges and consequently modifies the capillary force applied on the top grain. The 


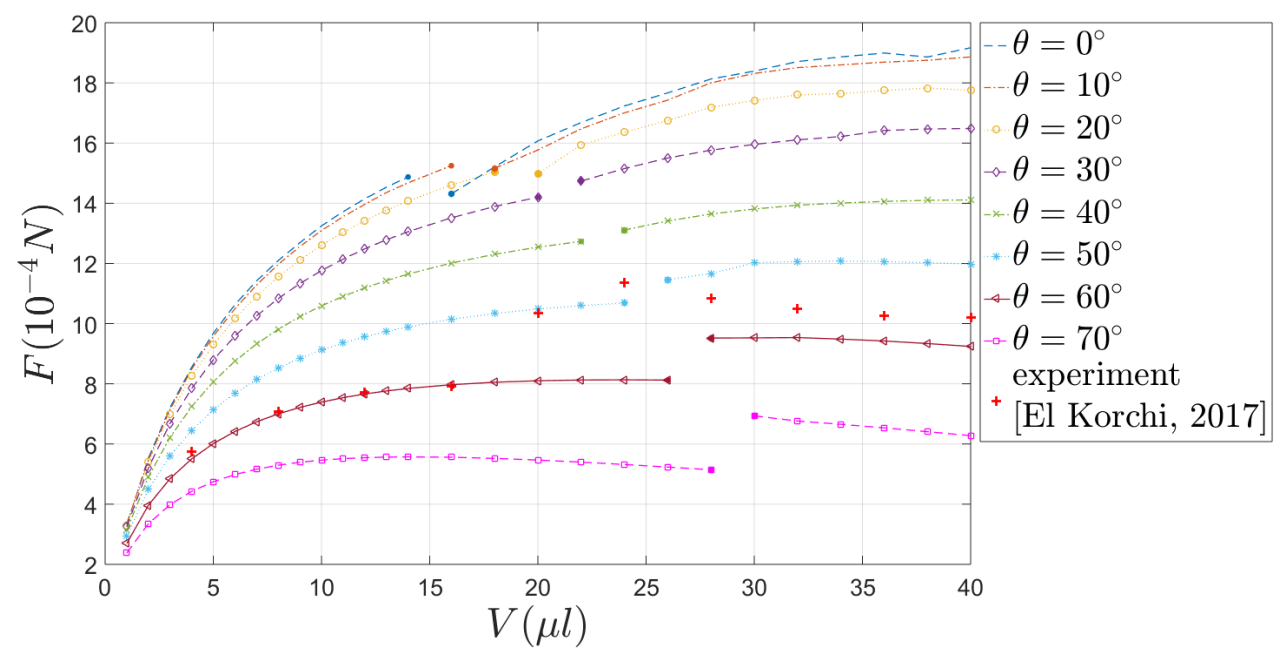

Fig. 9 Capillary force in a triplet of grains, as a function of the volume of water, calculated with Surface Evolver for $\gamma^{l g}=0.073 \mathrm{~N} . \mathrm{m}^{-1}$ and for different contact angles (dashed lines), and obtained experimentally in [37] (red plus symbols).

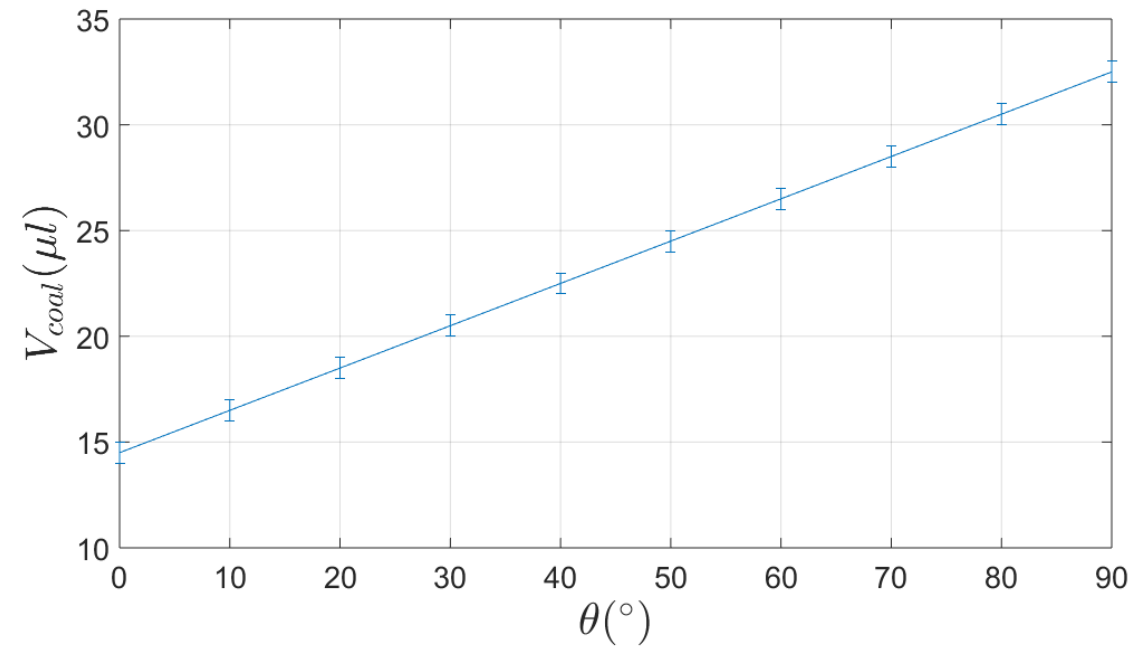

Fig. 10 Total volume of water at coalescence as a function of the contact angle. The solid line stands for the linear relationship: $V_{\text {coal }}=V_{\text {coal }}^{0}+\frac{\theta}{\Delta \theta} \Delta V_{\text {coal }}$ with $V_{\text {coal }}^{0}=14.5 \mu \mathrm{l}, \Delta V_{\text {coal }}=$ $18 \mu \mathrm{l}$ and $\Delta \theta=90^{\circ}$.

dimensionless Bond number can be introduced to compare gravitational effect to capillary forces in a system, apart from any water content consideration. A Bond number negligible with respect to 1 allows neglecting the gravity. Here, the Bond number given by the classical definition reads: 


$$
B o=\frac{r^{2} \Delta \rho g}{\gamma^{l g}}=2.14 \geqslant 1
$$

with $g=9.81 \mathrm{~m} . \mathrm{s}^{-2}$ the acceleration of gravity and $\Delta \rho=997 \mathrm{~kg} \cdot \mathrm{m}^{-3}$ the difference between liquid and gas density at $20^{\circ} \mathrm{C}$. Consequently, gravity cannot be neglected in the present case.

Fortunately, accounting for gravity is quite straightforward in our energy based approach as it simply consists in adding the potential energy term in the expression of the energy (in Eq. (4)) while keeping the minimization procedure unchanged.

The capillary forces in the assembly of three grains, with or without gravity, are plotted in Figure 11. As can be seen, gravity tends to decrease the capillary forces, as it is observed in [55], moving closer to the experimental data. On the contrary, the volume of coalescence remains almost the same, between $14 \mu \mathrm{l}$ and $14.5 \mu \mathrm{l}$, which means that the influence of gravity on the position of the triple line at the upper grain surface before coalescence is small.

It is worth noting that gravity increases the drop in capillary force at coalescence and affects more strongly the small volume cases in the subsequent coalesced regime. This can be explained by the shape of the liquid interfaces as illustrated in Figure 12 where is plotted the profile of the interfaces of a coalesced bridge in the plane formed by the centers of the grains, for $V=16 \mu \mathrm{l}$ and $V=30 \mu \mathrm{l}$ :

- At the top of the bridge, the displacement of a part of the water due to gravity decreases the radius of curvature of the meniscus contributing to the capillary force between the lower grains and the upper grain. As the capillary force tends to decrease with the radius of curvature [54], the contribution of the upper parts of the coalesced bridge to the vertical capillary force decreases. For the highest volumes, the displacement of the water in the upper part of the capillary bridge is relatively less important than for small volumes (a larger proportion of the water weight is supported by the bottom grains for large volumes, which limits water transfers), and the radius of curvature is less affected.

- At the bottom of the bridge, the displacement of the liquid/gas interface induces an increase in the vertical capillary force but with a smaller effect as the curvature is orthogonal to the force. Indeed, this portion of the capillary bridge contributes mainly to horizontal attraction between the bottom grains, 
which could not be measured with the experimental set up. The displacement of the liquid/gas interface at the bottom is larger for the highest volumes since the weight of the water bridge increases with its volume.

- In the end, as the displacement at the top of the bridge is less significant for higher volumes than for smaller ones, and more significant at the bottom, the impact of gravity on the vertical capillary force is consequently less important for the highest volumes.

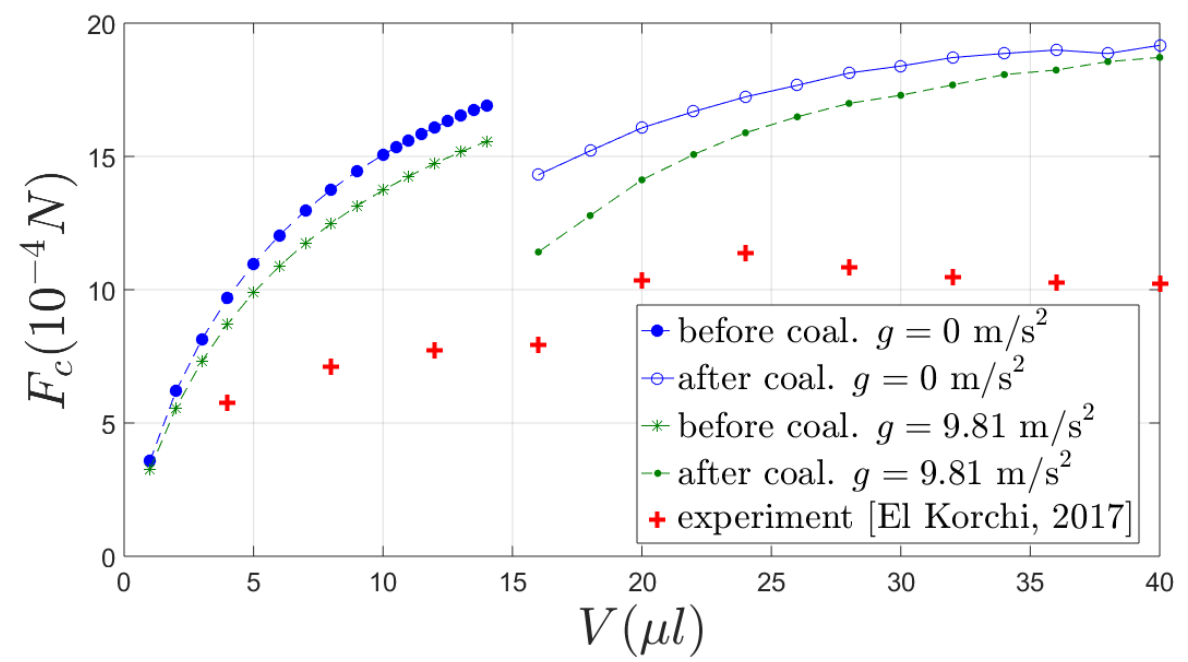

Fig. 11 Capillary force in a triplet of grains calculated numerically without gravity (blue circles) and with gravity (green stars and points), as a function of the volume of water, with $\gamma^{l g}=0.073 \mathrm{~N} \cdot \mathrm{m}^{-1}$ and $\theta=0^{\circ}$, and obtained experimentally in El Korchi et al. [37] (red plus symbols).

3.5 Influence of the surface tension of the liquid/gas interface

The value to be used for the liquid/gas surface tension is questionable since it was inferred but not directly measured in the experiments [37]. If the standard value $\gamma^{l g}=0.073 \mathrm{~N} . \mathrm{m}^{-1}$ corresponds to pure water in air at $20^{\circ} \mathrm{C}[52]$, water is known to be a polar liquid that easily captures impurities from the external environment, inducing substantial reduction in surface tension. In this context, a calibration of the surface tension value in a capillary bridge between two grains 

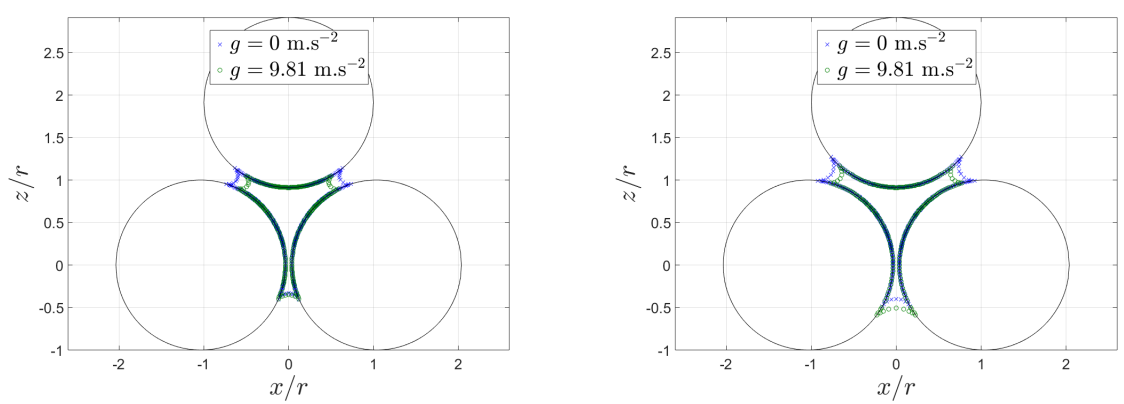

Fig. 12 Positions of the water interfaces obtained numerically in the plane formed by the centers of the grains for $V=16 \mu \mathrm{l}$ (left) and $V=30 \mu \mathrm{l}$ (right), without gravity (empty blue circles) and with gravity (green points).

was realized in [56] and shows that a value around $0.0693 \mathrm{~N} . \mathrm{m}^{-1}$ can reasonably be adopted. When gravity is not accounted for, surface energy and capillary force are basically proportional to surface tension. A decrease in surface tension induces a decrease in the same proportion for the capillary force, which may explain part of the discrepancy observed between the experimental and numerical curves in Figure 6.

When gravity is taken into account, a decrease in the surface tension will decrease proportionally the surface energy and will increase the relative contribution of gravity to the total energy of water. Thus, the decrease in the surface tension has a twofold effect on the capillary force decrease. Figure 13 shows the capillary forces before and after coalescence for the classical value $\gamma^{l g}=0.073 \mathrm{~N} \cdot \mathrm{m}^{-1}$ $(B o=2.14)$ and for the value from $[56] \gamma^{l g}=0.0693 \mathrm{~N} \cdot \mathrm{m}^{-1}(B o=2.26)$, with and without gravity, for $\theta=0^{\circ}$. The upper chart confirms that the dimensional capillary force decreases with gravity and decreasing surface tension. This observation is in agreement with the results from Murase et al. [55], which show that the vertical capillary force in a triplet decreases when Bond number increases. On the lower chart, the dimensionless capillary forces $F_{c}^{*}=\frac{F_{c}^{*}}{2 \pi r \gamma^{l g}}$ are plotted. It can been deduced from this chart that before coalescence, gravity and surface tension influence significantly and independently the capillary force. However, the relative importance of gravity over surface tension is not affected by the value of the surface tension. After coalescence, the differences between the dimensionless curves with gravity show that decreasing the value of $\gamma^{l g}$ increases the relative 
importance of gravity. Thus, the slight difference in the Bond number between the two cases is almost negligible before coalescence but becomes significant beyond coalescence.
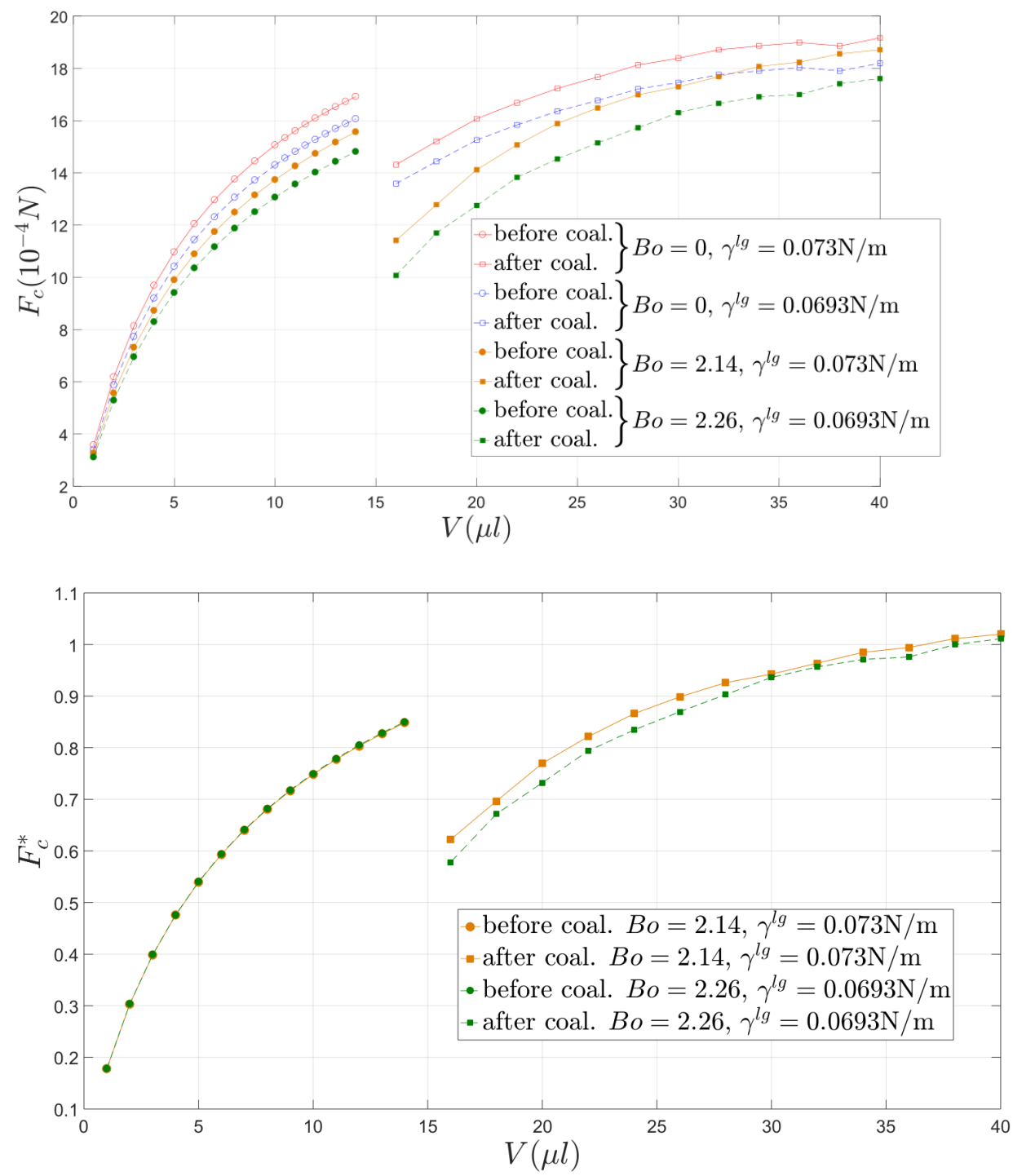

Fig. 13 Capillary force in a triplet of grains calculated numerically without gravity (empty symbols) and with gravity (full symbols), for $\gamma^{l g}=0.073 \mathrm{~N} / \mathrm{m}$ (solid line) and $\gamma^{l g}=0.073 \mathrm{~N} / \mathrm{m}$ (dashed line), with $\theta=0^{\circ}$, as a function of the volume of water. Upper: dimensional capillary forces, lower: dimensionless capillary forces. 
3.6 Calibration of the physical parameters

Investigating the influence of the physical parameters has pointed out that the vertical capillary force decreases with decreasing surface tension, increasing contact angle and addition of gravity. Consequently, accounting for gravity and using the more realistic value of the surface tension $\gamma^{l g}=0.0693 \mathrm{~N} . \mathrm{m}^{-1}[56]$, we investigate on a plausible range of contact angle values compatible with the experimental data by El Korchi et al. In Figure 14, the capillary forces have been plotted for two different values of the contact angle, when adding gravity, and with $\gamma^{l g}=$ $0.0693 \mathrm{~N} . \mathrm{m}^{-1}$. Before coalescence the numerical curve with $\theta=60^{\circ}$ matches quite well the experimental curve. The differences between the two curves tend to show that the contact angle is slightly lower for small volumes and a little higher just before coalescence. As regards the highest volumes, the capillary forces obtained numerically with $\theta=55^{\circ}$ are in good agreement with the experimental results. However, the fitted parameters should be considered with care as the comparison is made with a single experimental set.

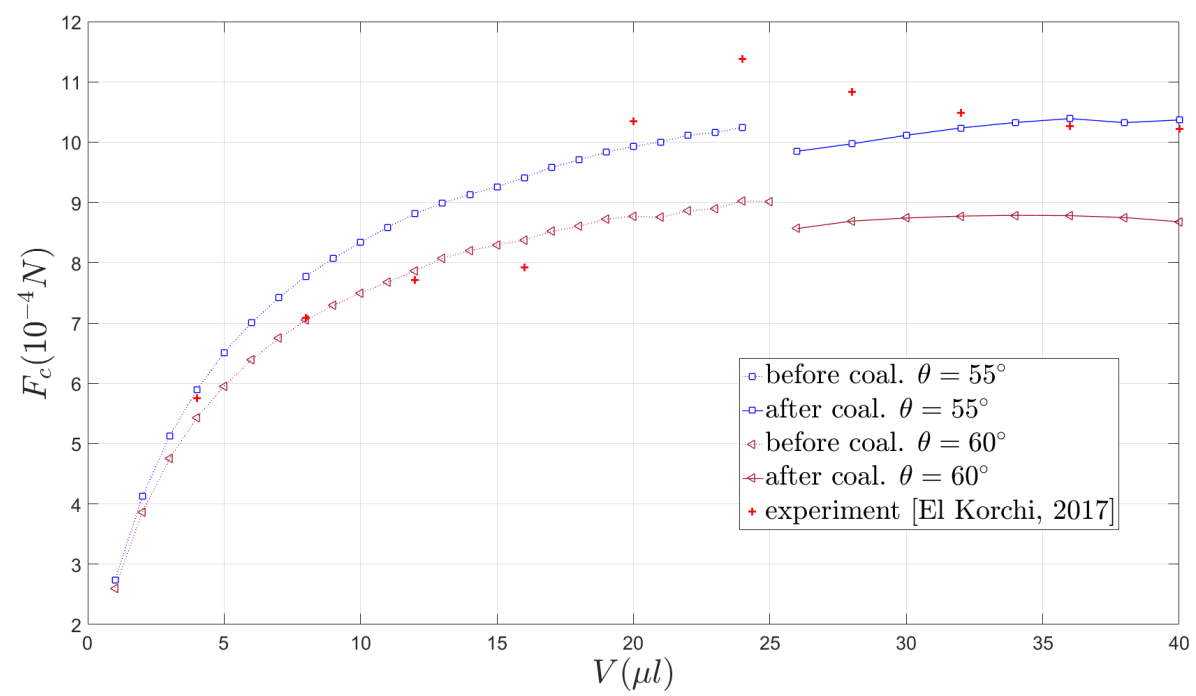

Fig. 14 Capillary force in a triplet of grains, as a function of the volume of water, calculated with Surface Evolver with $\gamma^{l g}=0.0693 \mathrm{~N} . \mathrm{m}^{-1}$ with $g=9.81 \mathrm{~m} . \mathrm{s}^{-2}, \theta=60^{\circ}$ (triangles) and $\theta=55^{\circ}$ (squares), before (dotted line) and after (solid line) coalescence, against experimental results from [37] (crosses). 
This range of contact angle values is in reasonable agreement with the experiment pictures (Figure 7 and El Korchi et al. [37]), where the contact angle varies roughly from $30^{\circ}$ to $60^{\circ}$. The picture tends to show that the contact angle is not the same on the upper and the lower beads, depending on the position of the triple line on each bead and then on the volume of water. At coalescence, the upper parts of the triple lines lie on areas previously wet, where a thin layer of water may remain. This leads to a contact angle different from the uncoalesced bridges and from the lower part of the coalesced bridge that took place on a previously dry surface, due to the hysteresis of the contact angle $[33,34]$. However, it should be underlined that the present formulation of the minimization problem in Surface Evolver does not allow for defining different contact angle values on the surface of a same given grain.

Depending on the contact angle value, coalescence now occurs for $V$ between $24 \mu \mathrm{l}$ and $26 \mu \mathrm{l}$, which is more than the experimental coalescence volume, in between 16 and $20 \mu \mathrm{l}$. Figure 15 shows that for $V=20 \mu \mathrm{l}$, the half-filling angle $\beta$ is less than $3^{\circ}$ under the value of the opening angle $\alpha$, which means that the two bridges are very close. It is therefore plausible that the contact could occur in practice for a smaller volume, due to some slight loss of symmetry, or to the presence of impurities in the water or at the surface of the beads.

As observed, this range of contact angle values provides satisfactory agreement between the numerical curve and the experimental data both before coalescence and after coalescence, but only for the highest volumes $(V \geqslant 32 \mu \mathrm{l})$. However, just after coalescence, for $V$ between $25 \mu \mathrm{l}$ and $32 \mu \mathrm{l}$, the calculated capillary forces are smaller than the measured ones.

Finally, Figure 16 depicts the geometry of the water interfaces with $\theta=55^{\circ}$ just after coalescence, for $V=26 \mu \mathrm{l}$, and later, for $V=36 \mu \mathrm{l}$. Obviously, the meniscus between the lowest spheres is still below the centers of the grains just after the coalescence, unlike the experiment. These results suggest that the energy minimization approach is able to reproduce the experimental observations before and far enough after coalescence. However, because of the differences observed at its vicinity, one could speculate on a transient state where the energy required to move the water to the configuration with the smallest energy is higher than the difference between the energy of the current geometry, and the smallest energy. 


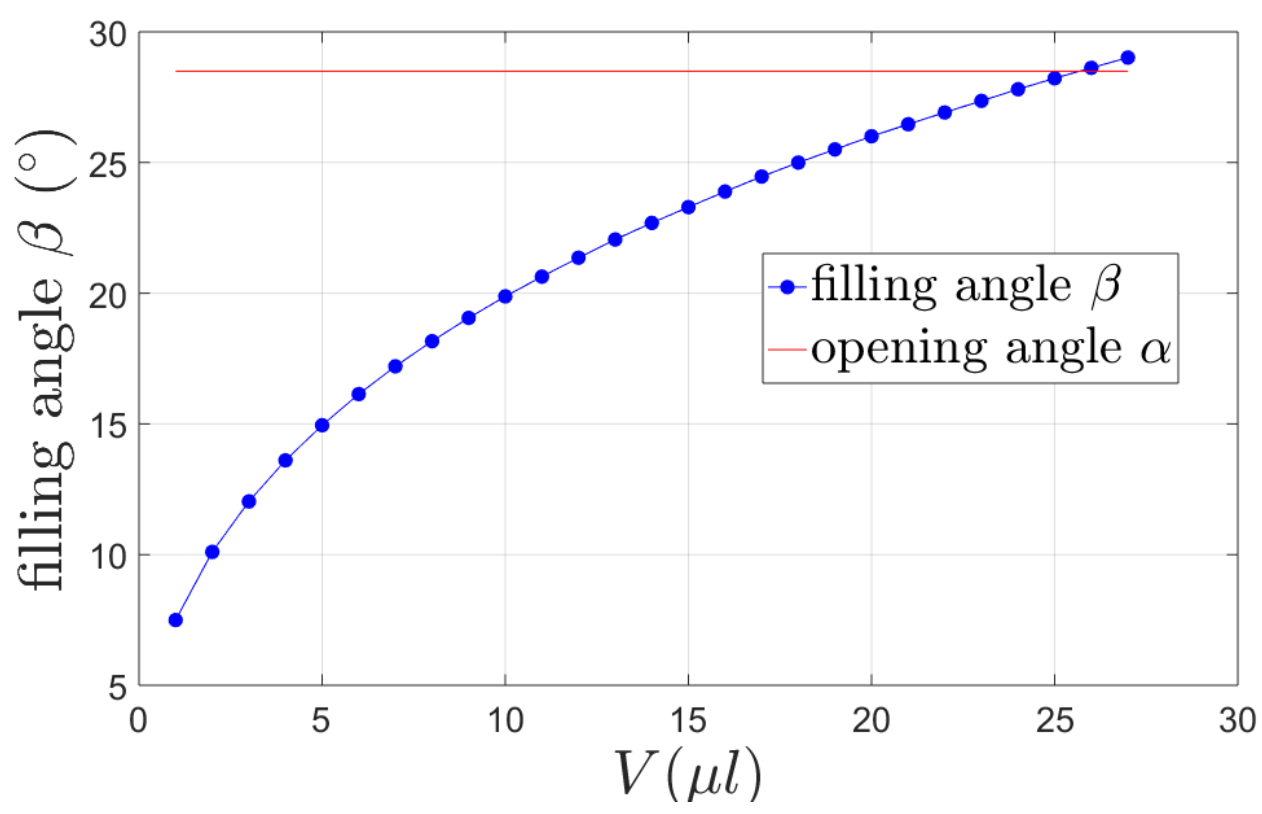

Fig. 15 Filling angle $\beta$ as a function of the volume of water in two uncoalesced bridges, obtained with $g=9.81 \mathrm{~m} . \mathrm{s}^{-2}, \gamma^{l g}=0.0693 \mathrm{~N} . \mathrm{m}^{-1}$ and $\theta=60^{\circ}$. The opening angle $\alpha$ characterizing the triplet geometry as shown in Figure 4 is marked as a horizontal solid line.

Moreover, dynamics effects on the surface tension value have been observed in

Hauner et al. [57]. It can lead to an important increase in the surface tension value around $0.09 \mathrm{~N} . \mathrm{m}^{-1}$, which implies an increase in the capillary force. As these effects occur at the atomic and molecular scales, the surface energy minimization method cannot reproduce this transient state.

\section{Concluding remarks}

To sum up this contribution, a numerical method based on surface energy minimization has been presented in order to estimate capillary forces in small assemblies composed of a few spherical grains. Firstly, the method has been challenged to model a single capillary bridge between two grains, in order to determine the evolution of the related capillary force as a function of both the intergranular distance and the volume of liquid. The results have been successfully compared with published experimental and numerical results. Secondly, the method was used to 


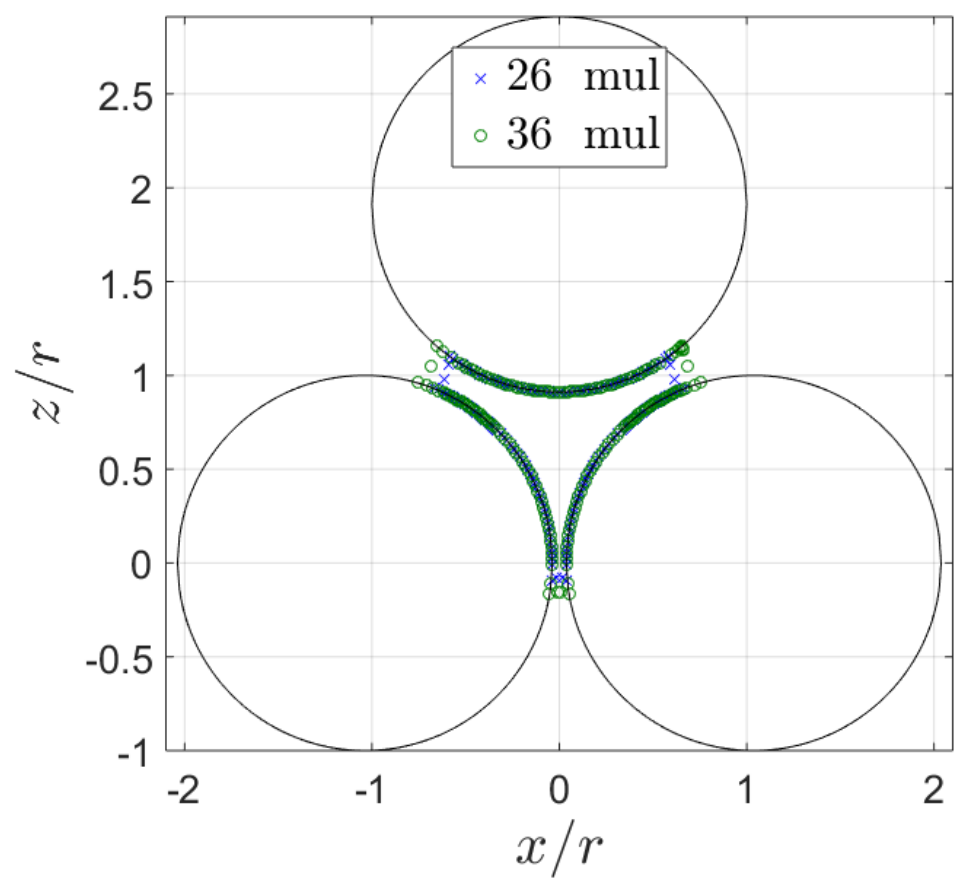

Fig. 16 Profile of water interfaces after coalescence, for $V=26 \mu$ l (blue crosses) and $V=36 \mu$ l (green open circles), with gravity and for $\gamma^{l g}=0.0693 \mathrm{~N} \cdot \mathrm{m}^{-1}$ and $\theta=55^{\circ}$.

investigate the evolution of capillary forces during coalescence of two capillary bridges in a triplet of spherical grains.

A comparison with published experimental results by El Korchi et al. [37] provides the opportunity to discuss the influence of physical parameters. The contact angle value was first investigated. It is shown to have a large influence on the capillary force, which decreases substantially when the contact angle increases. The contact angle has also an effect on the overall shape of the capillary force curve that can affect the evolution of capillary force after coalescence. It also has an important effect on the shape of the bridges, and a linear relationship between coalescence volume and contact angle has been found. Comparison with experiments shows that the contact angle depends on the volume of water and on the position of the triple line on the grain. Imperfections on the spheres surface are thought to influence the position of the triple line, leading to the hysteresis of contact angle. A plausible range of value has been found between $55^{\circ}$ and $60^{\circ}$. 
Considering the size of glass beads radius, gravity cannot be neglected. Gravity tends to decrease capillary force value. Its influence before coalescence is quite low, and the changes in coalescence volume is not significant. In the coalesced bridge, the influence of the gravity is more important. Paradoxically, the influence of gravity on capillary force is more important for the smallest volumes of the coalesced bridge, which can stem from the evolution of the morphology of the bridge with gravity.

Then, the influence of the surface tension has been investigated with and without gravity. In the absence of gravity, surface tension has a proportional effect on the capillary forces value, with no impact on the morphology of the bridge. However, when adding gravity, a decrease in the surface tension increases the Bond number value and then the relative contribution of the gravity in the total energy. The effects of the variation of the Bond number is quite negligible before coalescence but is more important after.

Finally, this sensitivity analysis gives a plausible range of values for the physical parameters. The present work underlines the relative influence of different physical parameters on the contact force value. It was found that the contact angle is the most important parameter to be calibrated to reproduce the experimental results. A convenient choice of contact angle should allow reproducing accurately experimental results before coalescence and sufficiently long after the coalescence. However, the coalescence of bridges is difficult to reproduce with a static method because of transient phenomena that are not governed by a simple energy criterion. The impossibility for the energy minimization software to account for spatial variations in the contact angle on the grain surfaces can also explain the remaining discrepancies between experimental and numerical data.

A reciprocal investigation of the rupture of liquid bridges in grains assemblies during drying will be considered in future work. By assuming that the rupture of a coalesced volume occurs when the energy of the non-coalesced configuration is lower than the energy of the coalesced configuration, it could be possible to predict the volume at rupture and the resulting evolution of capillary forces in drying with the presented numerical method. In addition, benefiting from the present approach to compute capillary forces in the case of well-defined geometry assemblies with small numbers of grains under a quasi-static loading, an extension to larger grain 
assemblies is currently in progress for potential enrichment of existing microme-

chanical and multiscale models, such as the H-model [47]. First results pertaining

to the pendular regime are about to be published [50].

Acknowledgements The authors acknowledge F. Rouyer for the helpful discussions on the Surface Evolver software. The authors also express their sincere thanks to the French Research Network GeoMech (GDRI CNRS) for promoting and favoring interactions between researchers. It helped improving the work by quality discussions.

\section{Compliance with ethical standards}

The authors declare that there is no conflict of interests regarding the publication of this article. Publication has been approved by all authors. None of the material presented in the paper is submitted or published elsewhere.

\section{References}

1. Hornbaker, D. J., Albert, R., Albert, I., Barabasi, A.-L. \& P., S. What keeps sandcastles standing? Nature 387, 765 (1997).

2. Pakpour, M., Habibi, M., Møller, P. \& Bonn, D. How to construct the perfect sandcastle. Scientific Reports 2, 549 (2012).

3. Terzaghi, K. The shearing resistance of saturated soils and the angle between the planes of shear. First international conference on soil Mechanics 1, 54-59 (1936).

4. Fredlund, D. G. \& Rahardjo, H. Soil mechanics for unsaturated soils (John Wiley \& Sons, Ltd, 1993).

5. Chalak, C. Multi-phase and multi-material interactions in granular media. Ph.D. thesis, Université Grenoble Alpes (2016).

6. Mitarai, N. \& Nori, F. Wet granular materials. Adv Phys 55, 1-45 (2006).

7. Badetti, M. et al. Rheology and microstructure of unsaturated wet granular materials: Experiments and simulations. Journal of Rheology 62, 1175-1186 (2018).

8. Scholtès, L., Nicot, B. C. F. \& Darve, F. Discrete modelling of capillary mechanisms in multi-phase granular media. Computer Modeling in Engineering and Sciences 297-318 (2009).

9. Duriez, J. \& Wan, R. Contact angle mechanical influence in wet granular soils. Acta Geotechnica 12, 67-83 (2017).

10. Delenne, J.-Y., Richefeu, V. \& Radjai, F. Liquid clustering and capillary pressure in granular media. Journal of Fluid Mechanics 762, R5 (2015). 
11. Richefeu, V., Radjai, F. \& Delenne, J.-Y. Lattice boltzmann modelling of liquid distribution in unsaturated granular media. Computers and Geotechnics 80, 353 - 359 (2016).

12. Montellá, E. P., Yuan, C., Chareyre, B. \& Gens, A. Hybrid multi-scale model for partially saturated media based on a pore network approach and lattice boltzmann method. Advances in Water Resources 144, 103709 (2020).

13. Bishop, A. W. T. \& Donald, I. B. Experimental study of partly saturated soil in the triaxial apparatus. In Proceedings of the 5th International Conference on Soil Mechanics and Foundation Engineering, vol. 1, 13-21 (Dunod, Paris, France, 1961).

14. Cuomo, S., Moscariello, M. \& Foresta, V. Simple shear tests on unsaturated soils. Procedia Engineering 158, 122-127 (2016).

15. Mason, G. \& Clark, W. C. Liquid bridges between spheres. Chemical Engineering Science 20, 859-866 (1965).

16. Willett, C. D., Adams, M. J., Johnson, S. A. \& Seville, J. P. K. Capillary bridges between two spherical bodies. Langmuir 16, 9396-9405 (2000).

17. Mielniczuk, B., Hueckel, T. \& El Youssoufi, M. S. Evaporation-induced evolution of the capillary force between two grains. Granular Matter 16, 815-828 (2014).

18. Pitois, O., Moucheront, P. \& Chateau, X. Liquid bridge between two moving spheres: an experimental study of viscosity effects. Journal of Colloid and Interface Science 231, 26-31 (2000).

19. Haines, W. B. Studies in the physical properties of soils: II. a note on the cohesion developed by capillary forces in an ideal soil. The Journal of Agricultural Science 15, 529-535 (1925).

20. Lian, G., Thornton, C. \& Adams, M. J. A theoretical study of the liquid bridge forces between two rigid spherical bodies. Journal of Colloid and Interface Science 161, 138 147 (1993).

21. Pepin, X., Rossetti, D., Iveson, S. M. \& Simons, S. J. R. Modeling the evolution and rupture of pendular liquid bridges in the presence of large wetting hysteresis. Journal of Colloid and Interface Science 232, 289 - 297 (2000).

22. Kruyt, N. P. \& Millet, O. An analytical theory for the capillary bridge force between spheres. Journal of Fluid Mechanics 812, 129-151 (2016).

23. Gagneux, G. \& Millet, O. Analytic calculation of capillary bridge properties deduced as an inverse problem from experimental data. Transport in Porous Media 105, 117-139 (2014).

24. Mielniczuk, B., Millet, O., Gagneux, G. \& El Youssoufi, M. S. Properties of pendular liquid bridges determined on delaunay's roulettes s. EPJ Web Conf. 140, 09042 (2017).

25. Nguyen, H. N. G., Millet, O. \& Gagneux, G. On the capillary bridge between spherical particles of unequal size: analytical and experimental approaches. Continuum Mechanics and Thermodynamics 31, 225-237 (2019).

26. Richefeu, V., Radjal, F. \& El Youssoufi, M. S. Stress transmission in wet granular materials. The European Physical Journal E 21, 359-369 (2006). 
27. Scholtès, L., Chareyre, B., Nicot, F. \& Darve, F. Micromechanics of granular materials with capillary effects. International Journal of Engineering Science 47, 64-75 (2009).

28. Gras, J.-P., Delenne, J.-Y., Soulié, F. \& El Youssoufi, M. S. Dem and experimental analysis of the water retention curve in polydisperse granular media. Powder Technology 208, 296 -300 (2011).

29. Gladkyy, A. \& Schwarze, R. Comparison of different capillary bridge models for application in the discrete element method. Granular Matter 16, 911-920 (2014).

30. Khamseh, S., Roux, J.-N. \& Chevoir, F. Flow of wet granular materials: A numerical study. Physical Review E 92, 022201 (2015).

31. Shen, Z., Jiang, M. \& Thornton, C. Shear strength of unsaturated granular soils: threedimensional discrete element analyses. Granular Matter 18, 37-49 (2016).

32. Badetti, M., Fall, A., Chevoir, F. \& Roux, J.-N. Shear strength of wet granular materials: Macroscopic cohesion and effective stress. The European Physical Journal E 41, 68-85 (2018).

33. Haines, W. B. Studies in the physical properties of soil. v. the hysteresis effect in capillary properties, and the modes of moisture distribution associated therewith. The Journal of Agricultural Science 20, 97-116 (1930).

34. Gao, L. \& McCarthy, T. J. Contact angle hysteresis explained. Langmuir 22, 6234-6237 (2006).

35. Semprebon, C., Scheel, M., Herminghaus, S., Seemann, R. \& Brinkmann, M. Liquid morphologies and capillary forces between three spherical beads. Phys. Rev. E 94 (2016).

36. Hueckel, T., Mielniczuk, B. \& Youssoufi, M. S. E. Micro-scale study of rupture in desiccating granular media. In Geo-Congress 2013, 808-817 (San Diego, United States, 2013).

37. El Korchi, F. Z., Jamin, F. \& El Youssoufi, M. S. Collapse of granular media subjected to wetting. EPJ Web Conf. 140, 10010 (2017).

38. Hueckel, T., Mielniczuk, B. \& El Youssoufi, M. S. Adhesion-force micro-scale study of desiccating granular material. Géotechnique 1-12 (2019).

39. Urso, M. E. D., Lawrence, C. J. \& Adams, M. J. Pendular, funicular and capillary bridges: results for two dimensions. Journal of colloid and Interface Science 220, 42-56 (1999).

40. Urso, M. E. D., Lawrence, C. J. \& Adams, M. J. A two-dimensional study of the rupture of funicular liquid bridges. Chemical Engineering Science 57, 677-692 (2002).

41. Gagneux, G. \& Millet, O. An analytical framework for evaluating the cohesion effects of coalescence between capillary bridges. Granular Matter 18, 16-28 (2016).

42. Rynhart, P. R., McLachlan, R., Jones, J. R. \& McKibbin, R. Solution of the younglaplace equation for three particles. Research Letters in the information and Mathematical Sciences 5, 119-127 (2003).

43. Wang, J.-P., Gallo, E., François, B., Gabrieli, F. \& Lambert, P. Capillary force and rupture of funicular liquid bridges between three spherical bodies. Powder Technology 305, 89-98 (2017).

44. Brakke, K. A. The surface evolver. Experimental Mathematics 1, 141-165 (1992). 
45. Broesch, D. J. \& Frechette, J. From concave to convex: Capillary bridges in slit pore geometry. Langmuir 28, 15548-15554 (2012).

46. Farmer, T. P. \& Bird, J. C. Asymmetric capillary bridges between contacting spheres. Journal of Colloid and Interface Science 454, 192-199 (2015).

47. Xiong, H., Nicot, F. \& Yin, Z. Y. A three-dimensional micromechanically based model. International Journal for Numerical and Analytical Methods in Geomechanics 41, 16691686 (2017).

48. Mielniczuk, B., Millet, O., Gagneux, G. \& El Youssoufi, M. S. Characterisation of pendular capillary bridges derived from experimental data using inverse problem method. Granular Matter 20, 14-24 (2018).

49. Yuan, Y. \& Lee, T. R. Contact Angle and Wetting Properties, 3-34 (Springer Berlin Heidelberg, Berlin, Heidelberg, 2013).

50. Xiong, H. et al. A novel multi-scale large deformation approach for modelling of granular collapse. Acta Geotechnica (2021).

51. Lambert, P., Chau, A., Delchambre, A. \& Régnier, S. Comparison between two capillary forces models. Langmuir 24, 3157-3163 (2008).

52. Molenkamp, F. \& Nazemi, A. H. Interactions between two rough spheres, water bridge and water vapour. Géotechnique 53, 255-264 (2003).

53. Scheel, M. et al. Morphological clues to wet granular pile stability. Nature Materials 7, 189-193 (2008).

54. Murase, K., Mochida, T. \& Sugama, H. Experimental and numerical studies on liquid bridge formed among three spheres. Granular Matter 6, 111-119 (2004).

55. Murase, K., Mochida, T., Sagawa, Y. \& Sugama, H. Estimation on the strength of a liquid bridge adhered to three spheres. Advanced Powder Technology 19, 349-367 (2008).

56. Nguyen, H. N. G., Zhao, C.-F., Millet, O. \& Gagneux, G. An original method for measuring liquid surface tension from capillary bridges between two equal-sized spherical particles. Powder Technology 363, 349-359 (2020).

57. Hauner, I. M., Deblais, A., Beattie, J. K., Kellay, H. \& Bonn, D. The dynamic surface tension of water. The Journal of Physical Chemistry Letters 8, 1599-1603 (2017).

\section{A Parametric study of numerical parameters in surface energy calculation with Surface Evolver}

Compared to the calculation for a bridge between two grains, the convergence of the surface energy minimization process is found to be more sensitive to the incremental distance $\delta d$ and to the numbers of gradient descent iterations and remeshings. The optimum calculation parameters are determined by a parametric study.

The first parameter to determine is the incremental distance $\delta d^{*}$ to apply to the system. The first criterion is to find a $\delta d^{*}$ not too large compared to the intergranular distances, but large enough to obtain a significant difference between the surface energies of the two 
configurations. The second criterion is to obtain a smooth variation of capillary forces with respect to the total volume of water contained in the cluster. In Figure 17, the capillary forces have been plotted as a function of the volume of water in funicular regime, for different value of $\delta d^{*}$ between $1.10^{-4}$ and $9.10^{-2}$. For $\delta d^{*} \leqslant 1.10^{-2}$, the values of capillary forces seem really imprecise, as the value of $\delta E_{s}$ is too small compared to the precision on $E_{s}$, which means that $\delta d^{*}$ requires being higher. For $\delta d^{*}>1.10^{-2}$, the impact of $\delta d^{*}$ on the capillary forces is less visible. In the following, we fix $\delta d^{*}=0.06$ as the curve obtained with this value presents the smoothest shape.

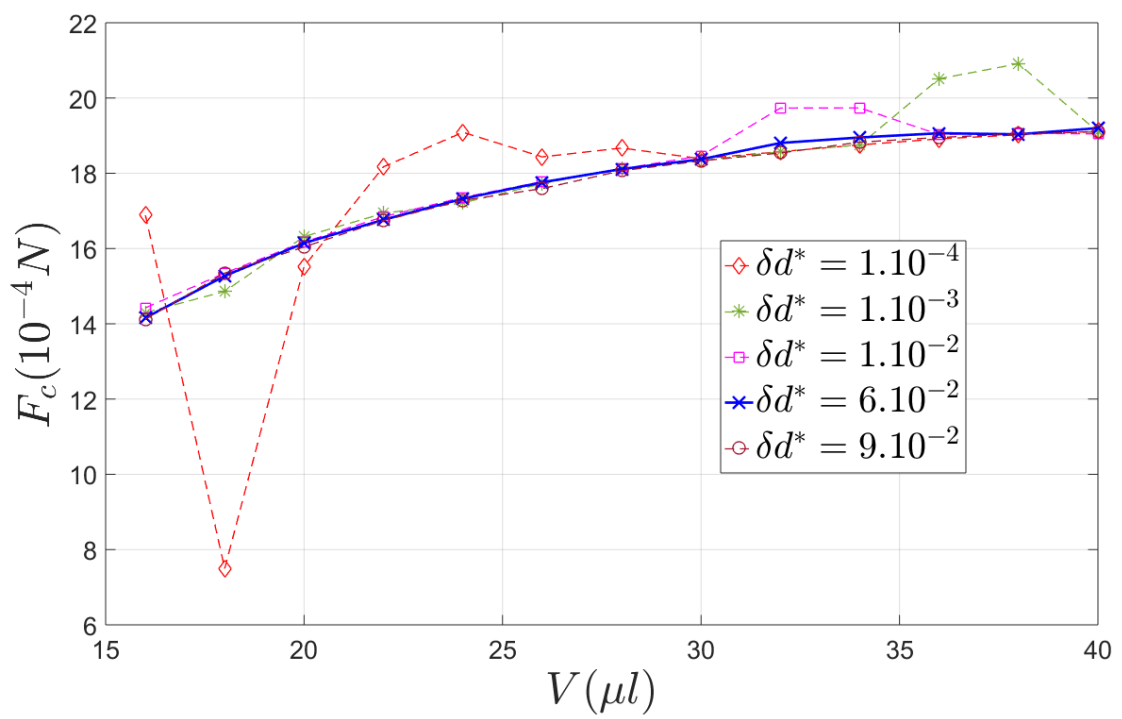

Fig. 17 Capillary force in a coalesced bridge between three grains, as a function of the volume of water, for different incremental intergranular distances $\delta d^{*}$, with 5 remeshings and 36 iterations between remeshings and after the last remeshing.

Sometimes, too much iterations of gradient descent method before a remeshing can lead to a divergence of the surface energy, when additional solid/gas interfaces are created outside the volume. Therefore, an optimized number of iterations between two remeshings has to be found. As shown in Figure 18, the number of iterations performed between remeshings affects the value of the capillary forces. We choose to perform 36 iterations between two remeshings since this curve is the smoothest.

Then, an optimized number of remeshings is determined from Figure 1. The capillary forces are calculated for a number of remeshings between 0 and 7 , in order to observe a convergence in Figure 19. Between 5 and 7 remeshings, we observe a maximal relative difference of $0.35 \%$, and a mean relative difference of $0.21 \%$. Between six and seven remeshings, we observe a maximal relative difference of $0.07 \%$ and a mean relative difference of $0.04 \%$. In the following, we perform six remeshings of the water interfaces. 


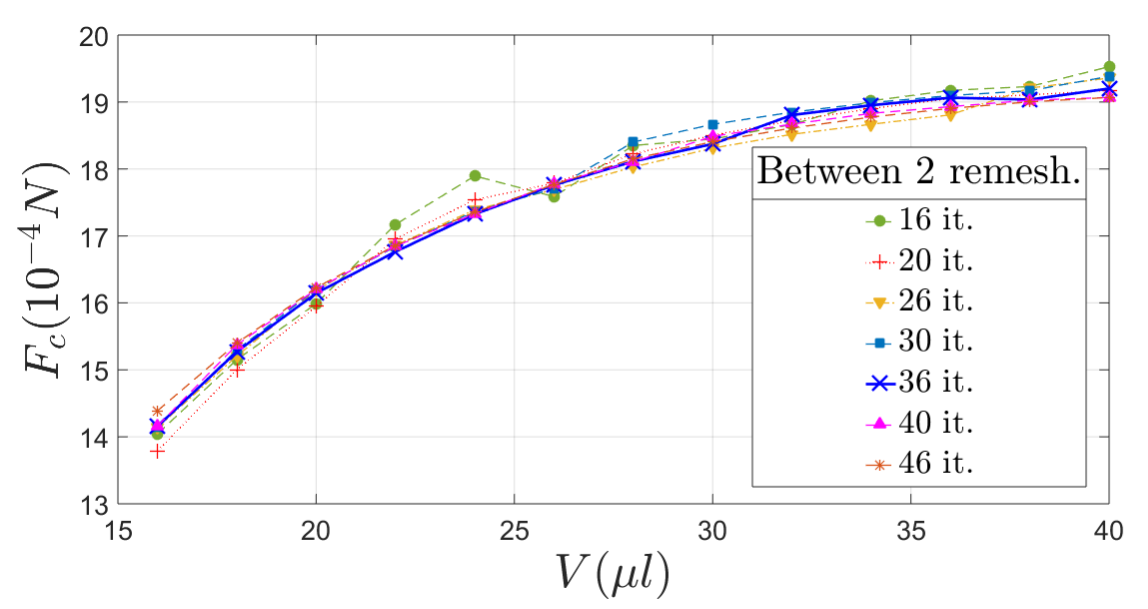

Fig. 18 Capillary force in a coalesced bridge between three grains, as a function of the volume of water, for different numbers of iterations between two remeshings, with $\delta d^{*}=0.06$.

\begin{tabular}{ll}
\hline$\delta d^{*}$ & 0.06 \\
\hline nb. of iterations between remeshings & 36 \\
\hline nb. of remeshings & 6 \\
\hline nb. of iterations after the last remeshing & 1400 \\
\hline
\end{tabular}

Table 1 Values of the calculation parameters used in a coalesced bridge in a triplet of grains with $\theta=0^{\circ}$

After the last remeshing, we have to control the convergence of the force calculation. Figure 20 shows the capillary force in the coalesced bridge as a function of the volume of water, for different numbers of iterations after the last remeshing. This curves show a convergence of the capillary force, as we observe a mean relative error of $0.05 \%$ between 1186 iterations and 1386 iterations, and of $0.02 \%$ between 1286 and 1386 iterations. In the following, we perform 1386 iterations after the last remeshing.

Finally, for a coalesced bridge between three grains, with a contact angle $\theta=0^{\circ}$, the following calculation parameters are chosen. 

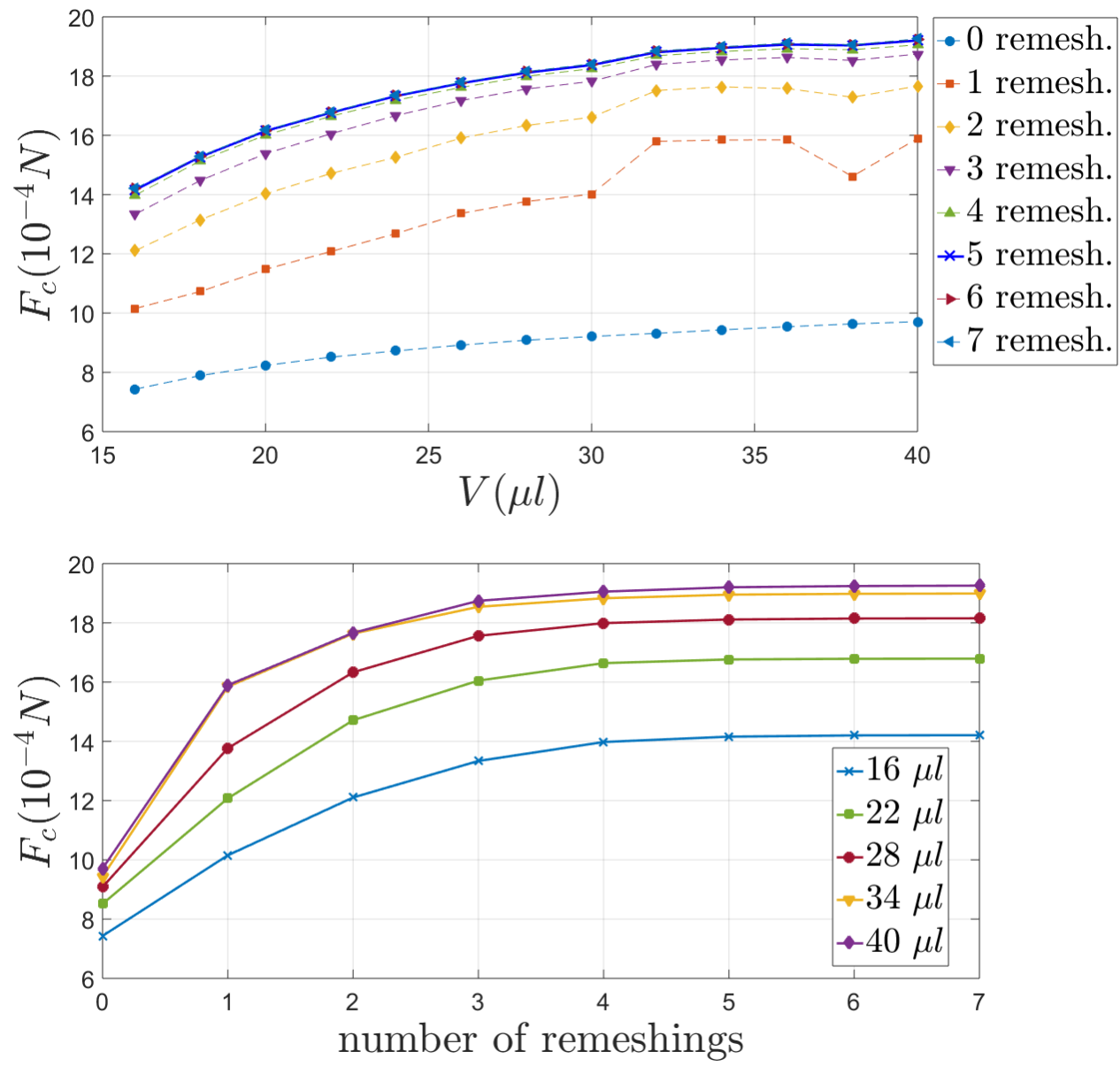

Fig. 19 Capillary force in a coalesced bridge between three grains, as a function of the volume of water, for different numbers of remeshings, (up), and as a function of the number of remeshings for different volumes of water (down), calculated with $\delta d^{*}=0.06$ and 36 iterations between two remeshings. 

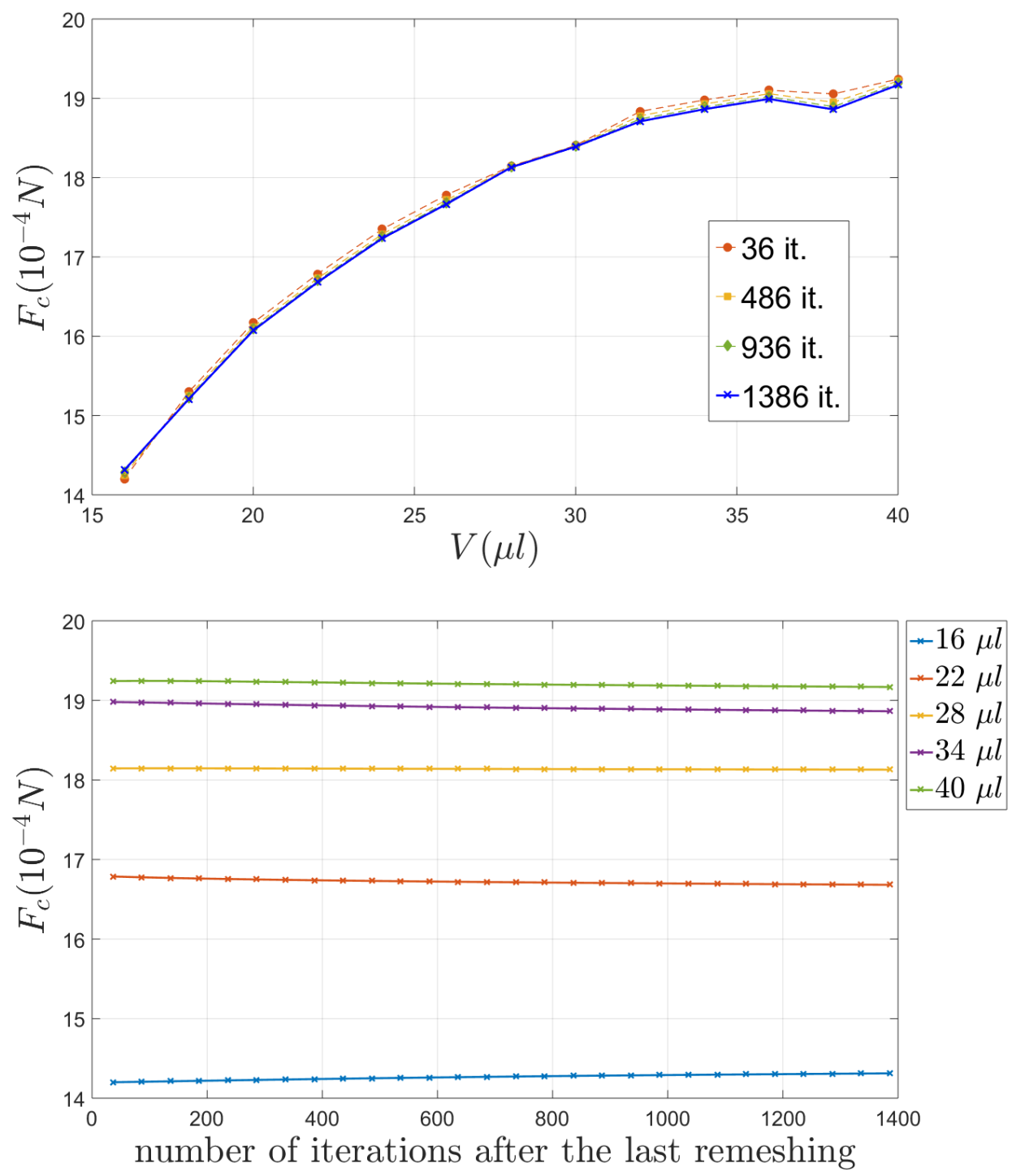

Fig. 20 Capillary force in a coalesced bridge between three grains, as a function of the volume of water, for different numbers of iterations after the last remeshing (up), and as a function of the number of iterations after the last remeshing for some volumes of water (down). 